\title{
Politiques d'emploi et de formation : innovations dans les territoires du Québec
}

\author{
Colette Bernier \\ Université Laval
}

\section{Introduction}

Дे l'ère de la globalisation, à côté des problèmes de chômage liés aux fermetures et aux délocalisations d'entreprises, les pénuries de maind'œuvre constituent un autre phénomène criant dans les territoires du Québec ${ }^{1}$. À partir d'une recherche menée récemment sur la formation de la main-d'œuvre dans les régions du Québec ${ }^{2}$, nous avons pu déceler l'émergence de stratégies inédites d'action publique concernant l'emploi et la formation face à ces problèmes de pénurie de main-d'œuvre ${ }^{3}$. La formation est alors utilisée par les acteurs du territoire comme outil pour mobiliser toutes les réserves de maind'œuvre disponibles pour l'emploi.

Souvent, ces stratégies d'action territoriale ont innové en débouchant sur de nouvelles perspectives de mobilité et de sécurité pour une main-d'œuvre dont les emplois se définissaient auparavant par leur extrême précarité. Ce faisant, elles ont même pu remettre en question un certain nombre de normes, par exemple en élargissant la relation d'emploi à plusieurs employeurs pour un même salarié. Par leur apparition simultanée dans plusieurs régions, on peut aussi se demander si ce type d'innovation ne représenterait pas plus qu'une réponse ponctuelle à une problématique locale et si elle ne rejoindrait pas des tendances plus lourdes d'évolution des politiques publiques. Avec la montée généralisée des emplois précaires, de telles expérimentations ne pourraient-elles pas en effet permettre de repenser les politiques publiques d'emploi et de formation en vue de contrer les effets de la précarisation?

C'est un tel questionnement qui guidera ce texte. Avant de présenter quelques-unes de ces expérimen- tations de l'action publique dans les territoires du Québec, nous discuterons des grandes tendances d'évolution de la relation d'emploi. Nous analyserons ensuite un certain nombre d'expérimentations territoriales autour de l'emploi et de la formation qui ont en commun de redéfinir la relation d'emploi traditionnelle. Nous montrerons ensuite comment ces diverses expérimentations peuvent être reliées à l'approche européenne des " marchés transitionnels de travail » et comment elles peuvent répondre à certains des enjeux actuels des politiques publiques d'emploi et de formation.

\section{Des changements à la relation d'emploi}

Dans les dernières décennies, plusieurs études ont constaté une crise du modèle de régulation, appelé « fordiste» en référence aux usines Ford où il a pris naissance. La norme du travail prédominante était alors celle d'un travail à temps plein, effectué par le chef de famille, sa vie durant, pour le même employeur. À partir de la crise des années 1980, différents changements dans le travail et l'emploi convergent vers une reconfiguration de cette norme.

Les entreprises se mettent alors à rechercher une plus grande flexibilité dans la production. En se restructurant, la grande entreprise redéfinit ses frontières autour d'alliances stratégiques, de sous-traitance, de fusions et d'acquisitions de toutes sortes. On assiste à une montée en importance de l'emploi dans les petites entreprises où se multiplient les formes d'emplois périphériques, sans protection. Par ailleurs, dans les plus grandes entreprises, une grande partie des réorganisations du travail se solde par une "précarisation de l'emploi », c'est-à-dire un affaiblissement du lien d'emploi. L'emploi type cesse d'être rattaché au mo- 
dèle «fordiste » de la grande entreprise avec protection de l'emploi et possibilités de promotions internes.

À côté de l'ancien modèle basé sur des garanties collectives à l'intérieur de la grande entreprise, un nouveau modèle a pris forme peu à peu dans les dernières décennies, celui de " l'employabilité ». Ce modèle repose sur l'idée que les salariés, maintenant appelés à changer d'employeurs plusieurs fois au cours de leur vie active, doivent se responsabiliser individuellement pour le maintien et le développement de leurs compétences, d'où l'importance donnée à la formation. De plus, dans ce modèle, «l'employabilité » repose désormais en grande partie sur le savoir-être et les comportements pour répondre aux impératifs d'implication exigés des nouveaux procès de travail.

Les politiques publiques d'emploi et d'éducation sont repensées en conséquence. Pour les clientèles de chômeurs et d'assistés sociaux, les politiques publiques mettent maintenant l'emphase sur les dispositifs « actifs » d'intégration à l'emploi dont la formation constitue l'outil par excellence. Les politiques de formation ne sont d'ailleurs pas en reste. Avec le modèle de «l'employabilité », la mobilité sociale est de plus en plus liée à la formation, et diverses mesures telles la reconnaissance des acquis extrascolaires, la promotion de congés de formation, l'ajustement des horaires de formation sont pensées pour favoriser l'investissement individuel à travers des politiques de «formation tout au long de la vie ».

Les microcosmes que constituent les régions nous semblent intéressants pour analyser l'ajustement de l'action publique aux nouvelles réalités du monde du travail. D'une part, plus que dans les grands centres, l'emploi dans les régions, par son caractère souvent saisonnier, est marqué par la précarité. D'autre part, c'est dans les territoires, au plus près des communautés, que les besoins sont le plus facilement révélés et que des projets trouvent la motivation pour l'action avec l'appui de la communauté. À travers les négociations qui entourent l'action publique dans certains projets territoriaux liant emploi et formation, nous avons en effet pu voir se profiler des ajustements aux politiques d'emploi et de formation qui pourraient apporter une plus grande sécurité aux individus face aux changements actuels de l'emploi. Après avoir examiné ces projets, nous montrerons, en dernière partie, comment ceux-ci rejoignent d'autres appro- ches récentes des politiques publiques d'emploi en Europe, en particulier l'approche des «marchés transitionnels de travail ».

\section{Des expériences liant formation, emploi et développement territorial}

Les projets que nous examinons ici ont tous été appuyés, sinon initiés, par les acteurs publics liés au développement régional. Ils sont ni plus ni moins le résultat du processus de déconcentration de l'action publique concernant les questions de l'emploi et de la formation qui s'est mis en place à la fin des années 1990 pour culminer en 2002 avec la tenue du Rendezvous national des régions. Ce processus a donné lieu, selon notre étude, à l'éclosion de nouveaux réseaux d'acteurs qui, autrefois, travaillaient en vase clos. À l'intérieur de ces réseaux, on retrouve maintenant, travaillant côte à côte, les bureaux régionaux des ministères touchant l'emploi, l'éducation et l'économie régionale avec les CRÉ (Conférence régionale des élus), et parfois les chambres de commerce ainsi que des représentants de la communauté.

\section{Un nouveau modèle a pris forme peu à peu dans les dernières décennies, celui de « l'employabilité ».}

Ces expériences ont en commun d'inscrire la formation dans des projets de développement territoriaux et de l'utiliser dans un objectif large de mobilisation/rétention des travailleurs déjà en emploi pour permettre la survie ou le développement d'industries locales ou régionales; en même temps, les expériences tendent à redéfinir la relation d'emploi non plus autour d'un employeur mais de plusieurs, parfois même autour du bassin régional d'emploi dans son ensemble, tout en proposant une plus grande mobilité et une meilleure sécurité d'emploi pour les travailleurs et travailleuses concernés.

L'élément déclencheur des projets est le plus souvent la constatation d'une pénurie de main-d'œuvre, réelle ou appréhendée, qui met en péril la survie des industries locales ou régionales, ou en entrave le développement. Aussi, les projets visent-ils d'abord la fidélisation de la main-d'œuvre à l'entreprise ou encore sa rétention dans la région. Ils s'adressent le plus souvent à des travailleurs précaires ${ }^{4}$, saisonniers ou à temps partiel à qui l'on offre de compléter leur temps 
de travail. La formation est toujours au cœur de ces projets; les pouvoirs publics structurent le processus et fournissent une aide financière aux entreprises et aux salariés. La formation professionnelle sert ainsi de «transition » pour les travailleurs déjà sur le marché du travail, soit pour les fidéliser, soit pour les préparer à une mobilité ou à de nouveaux emplois. Les activités sont organisées pour faire en sorte que les salariés exerçant leur emploi dans plusieurs entreprises de la région obtiennent une meilleure sécurité d'emploi et de meilleures perspectives de mobilité, donc une plus grande «employabilité ».

Il ressort de nos analyses deux cas de figures. Le premier concerne des entreprises individuelles appartenant le plus souvent à des secteurs d'emplois similaires; les expériences donneront lieu à des maillages d'entreprises qui se partageront des salariés précaires au sein d'une même région; l'objectif consiste à fidéliser la main-d'œuvre précaire en structurant sa mobilité. Le second cas de figure concerne plus largement un bassin régional d'emplois; il consiste à créer une réserve de travailleurs polyvalents et mobiles dans un bassin régional d'emploi comprenant plusieurs entreprises d'un même secteur d'emploi; l'objectif est ici de permettre aux entreprises de se donner une plus grande flexibilité et de s'assurer une maind'œuvre qualifiée.

\section{Il ressort de nos analyses deux cas de figures.}

\section{Premier cas de figure : fidéliser la main-d'oeuvre précaire en structurant sa mobilité}

Face à la pénurie de main-d'œuvre dans certains secteurs d'emplois où se trouvent une grande proportion d'employés précaires, saisonniers ou à temps partiel, un certain nombre d'actions vise à combler ces pénuries en structurant la mobilité de cette main-d'œuvre dans différentes entreprises tout en lui assurant une plus grande sécurité économique. La première expérience de ce type rencontrée lors de nos enquêtes avait été initiée par la Chambre de commerce d'une petite ville touristique des Laurentides. Recevant les plaintes des commerçants à l'effet qu'ils ne trouvaient plus la main-d'œuvre nécessaire à leur fonctionnement, la Chambre de commerce en vint à la conclusion, à la suite d'une étude portant sur ce problème, que le maillage d'emplois à temps partiel entre plu- sieurs commerces pour permettre aux entreprises d'avoir accès à un plus grand nombre de salariés et à ces derniers d'augmenter leurs heures de travail.

Les problèmes liés à la main-d'œuvre saisonnière ont aussi donné lieu à un maillage original d'entreprises en Gaspésie entre une usine de crabes et une usine de fabrication de couronnes de Noël. Cette expérience est le résultat du travail de différents ministères qui se sont réunis pour trouver des solutions aux problèmes de saisonnalité de la main-d'œuvre, entre autres par des projets de diversification. La plupart des usines des pêches fonctionnent entre six et sept mois par année, mais quatre usines dans le domaine du crabe ne permettaient pas aux travailleurs d'obtenir leurs prestations de chômage. Le même problème de précarité était vécu dans une usine de fabrication de couronnes de Noël de la région dont la pointe de production se situait autour des Fêtes. Face à un projet d'agrandissement de l'usine, on craignait par ailleurs de ne pas trouver la main-d'œuvre nécessaire. De là l'idée d'une collaboration avec une usine de crabes de la région qui cherchait aussi à stabiliser sa main-d'oeuvre. Une formation a été donnée, avec l'aide d'EmploiQuébec, pour les 200 travailleurs et travailleuses de l'usine de crabes par 16 employés de l'usine de couronnes de Noël. Suite à cette expérience, l'usine de couronnes prévoit se diversifier davantage par l'assemblage de pales d'éoliennes avec l'arrivée de cette industrie dans la région. L'histoire est à suivre en ce qui concerne les conditions de travail et la sécurité d'emploi des salariés.

Une expérience similaire a eu lieu dans un méga complexe de villégiature des Laurentides. S'il s'agit ici d'une seule entreprise, la division du travail entre secteurs sur une base saisonnière peut l'apparenter à un ensemble d'entreprises. Au plus fort de la saison hivernale, l'entreprise compte plus de 2000 employés et draine donc le bassin régional de main-d'œuvre. Bien que l'entreprise attire de nombreux travailleurs et travailleuses, elle doit aussi composer avec des pénuries à certains postes (du fait de l'éloignement et des conditions de travail) et, pour cette raison, a dû penser à une politique de «fidélisation » de sa maind'œuvre. Ainsi, l'été, lorsqu'il y a moins d'achalandage et qu'il y a des mises à pied temporaires, l'entreprise donne la priorité à ses employés de la saison hivernale. Fruit d'une négociation patronale-syndicale, on a de plus mis sur pied une banque de salariés polyvalents dont l'objectif est de stabiliser les 
emplois : «Plutôt que d'avoir des employés qui travaillent à mi-temps, on va essayer d'avoir un employé qui travaille à plein temps en jumelant des postes, maximiser les heures de travail de nos travailleurs en place plutôt que d'aller recruter ", avance la direction des ressources humaines. De plus, l'entreprise se sert de la formation (surtout en anglais langue seconde) pour retenir la main-d'œuvre jusqu'à l'hiver, la fidéliser. On fait d'abord appel aux employés temporairement mis à pied qui reçoivent alors pour se former des prestations d'assurance-emploi et un complément de revenu d'Emploi-Québec et éventuellement des frais de déplacement et de garderie.

Outre le fait, dans les deux cas, de jumeler des entreprises ou des services pour faire face aux pénuries de main-d'œuvre et en même temps permettre une plus grande régularité de travail aux salariés, la dernière expérience innove encore en considérant la formation en période de chômage comme une activité faisant partie quasi intégrante de l'emploi. Nous verrons plus loin comment nous pouvons relier ce type d'expérience à l'approche des "marchés transitionnels de travail » qui voudrait à la fois assurer une plus grande flexibilité aux entreprises et une plus grande sécurité d'emploi aux salariés. La dernière expérience, par le nombre de salariés impliqués, s'apparente aussi au prochain cas de figure où les actions concernent, de façon plus large que celles du premier cas de figure, des bassins régionaux d'emploi.

\section{Deuxième cas de figure : former des travailleurs qualifiés pour un bassin régional d'emploi}

Ces autres expérimentations ont eu lieu dans des régions, la plupart du temps là où prédomine un secteur d'emploi, soit pour augmenter la flexibilité des entreprises en formant des travailleurs qualifiés et polyvalents, soit pour faire face à des pénuries réelles ou appréhendées de main-d'œuvre. Ici encore, l'idée est d'assurer à la fois une plus grande flexibilité aux entreprises et une meilleure sécurité d'emploi aux salariés, en se servant entre autres de la formation.

La région de la Mauricie, où se retrouve le secteur traditionnel du textile, a été très active pour trouver des solutions aux problèmes de pénurie de maind'œuvre. Ainsi, à Louiseville, le Centre local d'emploi, avec la collaboration des entreprises du secteur du textile, ont mis en place une équipe polyvalente de travailleurs et travailleuses à la disposition de plu- sieurs entreprises de la localité. Dans ce secteur, la main-d'œuvre est vieillissante et il est difficile d'intéresser les jeunes à s'y diriger à cause de conditions de travail difficiles. À partir d'une liste de rappel utilisée par une des entreprises pour combler ses absences, Emploi-Québec a formé ces travailleurs et travailleuses sur l'ensemble des opérations liées au métier pour rendre ces personnes polyvalentes, leur permettant de faire du remplacement dans plusieurs entreprises de la région.

La région de la Gaspésie-Les-Îles est aussi à l'origine d'un projet intéressant alliant le développement de l'industrie éolienne sur son territoire et la hausse du niveau de scolarité de sa population. Dans un contexte de crise, le Plan de relance et de diversification de l'économie de 1999 a été l'occasion de mettre de l'avant le développement de l'énergie éolienne. Lors des rencontres entourant le plan, un élément choc a alors catapulté la formation au premier rang des préoccupations lorsqu'on s'est aperçu, chiffres à l'appui, que la main-d'œuvre compétente ferait terriblement défaut dans l'éventualité du développement attendu. À la suite d'une Journée de réflexion, organisée en décembre 2001 et où se retrouvaient plus de 150 partenaires de différents milieux socio-économiques, diverses actions de formation furent proposées. Un des projets qui rejoint notre propos concerne une nouvelle mesure de formation mise en place par Emploi-Québec qui sera ajoutée à un programme existant d'emplois temporaires permettant aux travailleurs de se qualifier pour l'assurance-emploi. Ainsi, à partir de 2002, les 45 ans et moins qui n'avaient pas leur secondaire $\mathrm{V}$ et qui voulaient avoir accès au programme d'emplois temporaires pour se qualifier à l'assurance-emploi devaient s'engager à se former. En conséquence, plus de 500 adultes âgés entre 18 et 45 ans, en général des travailleurs et travailleuses des usines de transformation de poissons, sont retournés en formation générale dans le cadre des cours aux adultes des commissions scolaires de la région.

Ce type de mesures correspond assez bien à l'approche des "marchés transitionnels » qui, comme on le verra, vise à ce que diverses activités en marge de l'emploi (formation, congés parentaux, pré-retraite, etc.) puissent être associées à une garantie de rémunération et constituer des passerelles vers d'autres positions sur le marché du travail pour éventuellement renouveler les bases de la sécurité économique des salariés. 
En 2001, la Table métropolitaine (relevant d'EmploiQuébec) a également développé un projet dans le secteur du bioalimentaire. Ce dernier consistait en une offre de formation concertée avec les maisons d'enseignement des cinq régions de la Région métropolitaine de recensement (RMR). Les entreprises identifiées dans le projet pilote sont, pour quatre d'entre elles, des PME du secteur de la transformation et une PME du commerce en alimentation. Le réseau des maisons d'enseignement a pour objectif de développer des projets pilotes de formation en entreprise visant à mettre en place des formations transférables d'une entreprise à l'autre. Les formations se donnent autant que possible dans l'entreprise et sur des périodes très courtes pour « intégrer la formation à la semaine de travail ». Dans les suites de ce projet, une des cinq régions a mis de l'avant l'idée d'utiliser les travailleurs et travailleuses de la région ainsi devenus polyvalents pour des remplacements dans différentes usines de la région.

Ainsi, à l'analyse, plusieurs projets territoriaux de formation remettent radicalement en question l'ancien modèle de la relation d'emploi pensé autour de la grande entreprise offrant sécurité d'emploi et possibilités de promotion. En remplacement de ce modèle, le regroupement de PME autour d'échanges de salariés et de formations paraît désormais une solution envisageable pour offrir une certaine flexibilité aux entreprises en même temps que mobilité et sécurité d'emploi aux travailleurs. La simultanéité des expérimentations dans différentes régions et l'appui, si ce n'est le rôle central des pouvoirs publics dans ces expériences, montrent qu'il ne saurait s'agir de cas isolés, mais bien plus de tendances probables d'évolution. Enfin, le fait que de telles expériences soient aussi mises de l'avant dans certaines approches européennes, telle l'approche des marchés transitionnels de travail, ouvre la voie à des avancées en ce sens au Québec.

\section{Au total, on veut mettre de l'avant un « modèle social solidaire pour le siècle qui commence $»$.}

\section{L'approche des « marchés transitionnels du travail »}

L'approche des marchés transitionnels trouve son origine dans divers rapports gouvernementaux (notam- ment les rapports Boissonat ${ }^{5}$ et Supiot $^{6}$ en France) qui tentent d'élargir l'assise de la protection sociale en prenant en compte non seulement l'activité professionnelle rémunérée dans un emploi régulier, mais plus généralement la participation à des activités sociales utiles ou jugées souhaitables. Ainsi, avec cette approche, il s'agirait de stabiliser la main-d'œuvre sur une base élargie et assouplie et de donner de nouveaux droits aux travailleurs et travailleuses en structurant leurs perspectives de mobilité. L'approche s'inspire de nombreux acquis obtenus dans les pays de l'Europe du Nord, notamment les Pays-Bas, la Suède et le Danemark.

Cette approche a été développée dans les années 1990 dans le cadre d'un programme international financé par la Communauté européenne auprès de dix équipes nationales de recherche ${ }^{7}$. Le pari que font les tenants de cette approche est que de telles politiques pourraient concilier la flexibilité recherchée par les entreprises sur le marché du travail avec la sécurité recherchée par les salariés et les syndicats en organisant ce que certains appellent une «flexicurité ${ }^{8}$ ». Au total, on veut mettre de l'avant une vision alternative au modèle américain, ou pour reprendre les termes de Gazier (2003), un «modèle social solidaire pour le siècle qui commence $»$.

Dans cette approche, la participation à des activités sociales, utiles ou jugées souhaitables, est considérée comme une transition par rapport au marché du travail, d'où le terme "marchés situation de référence constituée par l'emploi et comprennent cinq champs principaux : 1) les transitions au sein même de l'emploi; 2) entre l'emploi et le système éducatif; 3) entre l'emploi et le chômage; 4) entre l'emploi et les activités domestiques et 5) entre l'emploi et la retraite. Elles peuvent être illustrées comme dans la figure 1.

L'approche des "marchés transitionnels » a l'avantage de systématiser les transitions entre les activités de travail et les autres activités en donnant de nouveaux droits aux travailleurs. Ces différentes activités seraient en effet associées à une garantie de rémunération (cofinancement) sur la base d'une négociation. En constituant des passerelles vers d'autres positions sur le marché du travail, on renouvellerait ainsi les bases de la sécurité économique des salariés.

C'est parce que les marchés transitionnels constituent des projets négociés qu'on les retrouvera plus souvent 
aux niveaux local et régional, plus près des intervenants. Pourtant, il ne faut pas se leurrer, de telles actions pourraient rester marginales si elles ne peuvent s'inscrire dans de nouvelles règles. Aussi, selon Gazier', même si l'essentiel des discussions se passe à un niveau local ou régional, il y a nécessité d'un cadre national encadrant l'organisation des marchés transitionnels. Pour cette raison, nous nous demanderons, pour terminer, dans quelle mesure de tels projets sont compatibles avec les relations du travail au Québec et quels types de changements l'adoption de telles politiques demanderaient aux acteurs syndicaux.

Figure 1 - Les cinq principaux champs des marchés transitionnels

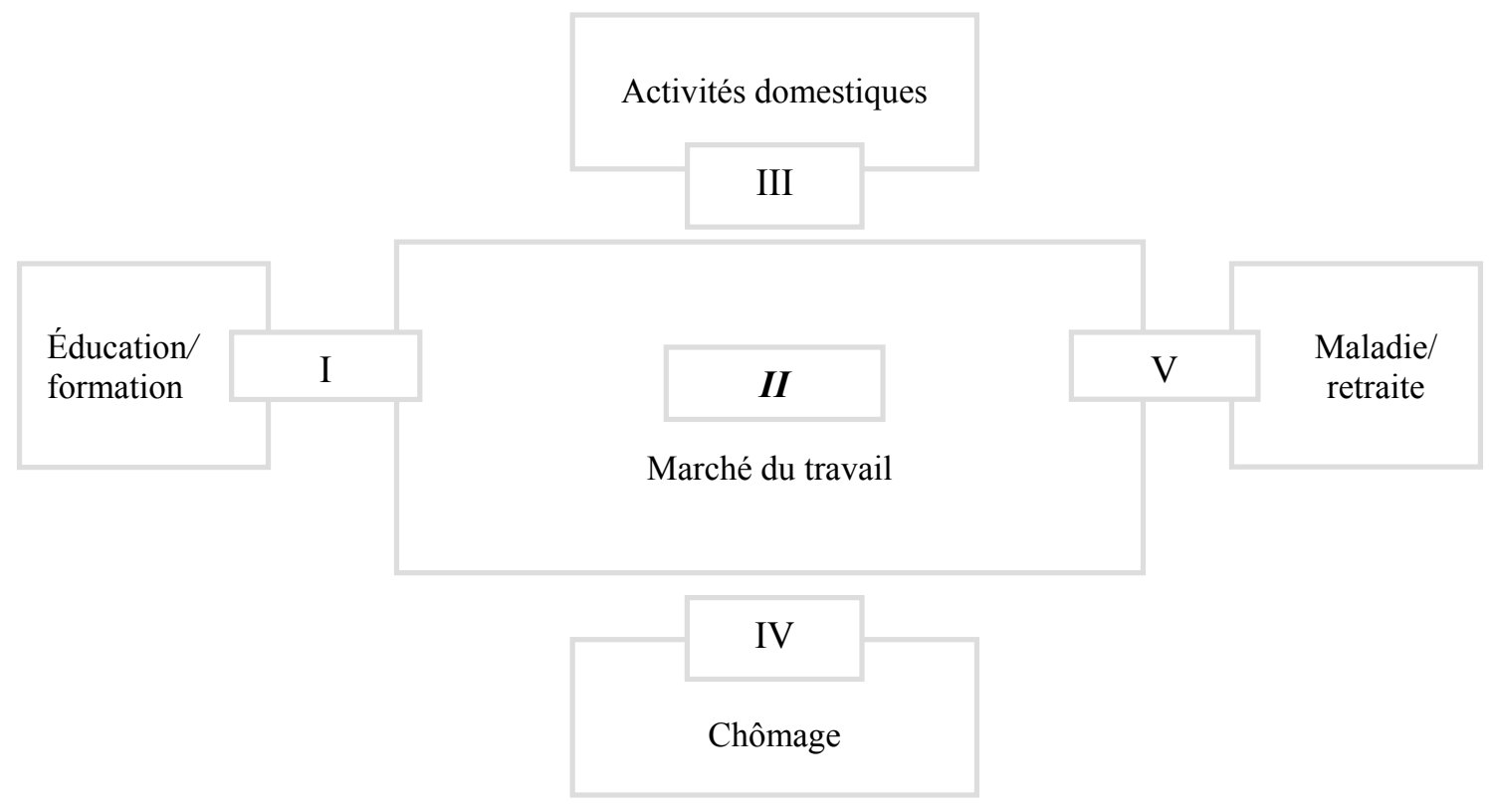

Source : Repris de Schmid, G. (2002).

\section{La nécessité de repenser les modes traditionnels de représentation et d'action syndicale}

Pour aller dans le sens de telles politiques, cela demanderait un certain nombre d'ajustements aux syndicats nord-américains. Ces ajustements concernent autant les niveaux de représentation, de mobilisation et d'action syndicale que les alliances nécessaires avec d'autres groupes sociaux. Nous allons le voir, l'action syndicale va déjà en ce sens.

Au Québec, comme dans l'ensemble de l'Amérique du Nord, les unités d'accréditation et de négociation syndicales sont formées sur la base d'entreprises individuelles et très rarement sur une base sectorielle ou de branche, comme c'est souvent le cas en Europe. Mais, l'entreprise n'est peut-être plus le niveau adéquat de représentation face aux nombreuses transitions d'emploi qui risquent de marquer la vie des salariés, à l'avenir. Ainsi, constatant un déséquilibre entre les politiques traditionnelles du marché du travail et les changements actuels à l'emploi, des experts américains ${ }^{10}$ proposent un certain nombre de mesures aux syndicats pour tenter de représenter les travailleurs non plus à partir d'une activité chez un employeur à un moment donné, mais durant toute leur vie active. Ils avancent que, si les syndicats doivent continuer à servir leurs membres traditionnels, il leur faut aussi se préoccuper des travailleurs en dehors du noyau central et s'occuper des besoins des travailleurs précaires et de ceux à carrières multiples. Pour cela, il leur faut entrevoir leur action en termes de la vie active de leurs membres durant laquelle les syndicats devraient offrir des services pour maintenir l'employabilité et la mobilité de leurs membres.

Pour ce, il faut élargir la base de la mobilisation à un ensemble d'organisations reliées au monde du travail et de l'éducation. Ainsi, selon ces mêmes auteurs, à côté des acteurs traditionnels du monde du travail, les syndicats doivent reconnaître l'émergence, durant 
les années récentes, de nouveaux acteurs et former des coalitions avec ces groupes, qu'il s'agisse de groupes communautaires, des réseaux de l'éducation et de la formation (entre autres d'apprentissage tout au long de la vie), d'organismes de recrutement de travailleurs temporaires ou d'autres organisations de partage du travail. Par ailleurs, en suivant ce raisonnement, la perspective des marchés transitionnels pourrait permettre d'élargir les publics cibles de la négociation et mettre de l'avant des actions conjointes, de nouvelles solidarités avec des groupes à la marge de l'emploi, chômeurs, assistés sociaux ou encore travailleurs précaires.

\section{Avec l'approche alternative des marchés transitionnels, il ne s'agit certainement pas d'accepter la tendance à la précarisation des emplois, mais plutôt d'inventer de nouvelles mesures de sécurité sur la base des réalités actuelles du marché du travail et de l'emploi.}

Au Québec, certaines expériences allant dans le sens d'une représentation des salariés au-delà de l'entreprise ont déjà été tentées. Par exemple, dans les années 1990, des tentatives faites par la Confédération des syndicats nationaux de revoir le Code du travail pour permettre la syndicalisation sur une base individuelle n'ont pas abouti. Pas plus d'ailleurs que des revendications déjà anciennes concernant un changement au Code du travail pour ouvrir la voie à la syndicalisation et à la négociation multi patronale, éventuellement sur une base sectorielle ou encore dans un bassin régional d'emploi. Dans les débats syndicaux de la dernière décennie au Québec, alors que l'établissement de liens solides avec ces nouveaux acteurs en émergence est déjà en marche, la question de l'action syndicale aux niveaux local et régional est aussi de plus en plus à l'ordre du jour.

De plus, pour les syndicats québécois, la période actuelle de lutte contre la privatisation des services publics et la précarisation des emplois pourrait leur fournir l'occasion de s'imposer dans la négociation des questions de l'organisation du travail et de la formation. Et ce, d'autant plus que l'approche des marchés transitionnels leur donne les outils pour négocier les questions de qualification, de mobilité, de carrière et de formation dans une approche globale du pro- blème et donc d'ouvrir de nouveaux champs de négociation. Les marchés transitionnels ne consistent-ils pas, pour reprendre les termes de Gazier (2003), « en des négociations collectives enrichies, décentralisées, qui associent de nouveaux partenaires et recherchent de nouveaux financements »?

\section{Conclusion}

Si plusieurs des évolutions actuelles de l'emploi et du travail peuvent assombrir les perspectives concernant l'emploi dans les régions, certaines expérimentations actuelles ouvrent la voie à l'action publique pour repenser la sécurité des salariés sur des bases nouvelles. Parmi celles-ci, l'essentiel est sans doute l'idée de normes inédites en train de se dessiner autour des transitions entre différentes activités (travail, activités domestiques, formation, etc.). Ce qui se joue en filigrane dans les actions publiques analysées, c'est une remise en question des politiques traditionnelles de l'emploi basées sur une vision linéaire et rigide des « plans de vie » allant des études à la vie active et à la retraite pour aller vers un modèle plus flexible qui offre la possibilité de distribuer sur l'ensemble de la vie, de façon souple, les périodes de travail, d'études, d'activités domestiques, de loisir et de retraite ${ }^{11}$.

Pour pouvoir penser de telles politiques à plus long terme, il faut d'abord comprendre que l'époque actuelle en est une de reconfiguration de la relation d'emploi traditionnelle. L'ancien modèle fordiste construit autour de «marchés internes de travail » offrant des perspectives de mobilité et une sécurité d'emploi sur la base de l'ancienneté dans la grande entreprise est affaire du passé. Pour ceux et celles qui pourraient avoir la nostalgie de ce modèle, il ne faut pas oublier qu'il ne s'appliquait qu'à une partie seulement de la main-d'œuvre active, une main-d'œuvre privilégiée dans un marché du travail segmenté parfois à l'extrême. Par ailleurs, avec l'approche alternative des marchés transitionnels, il ne s'agit certainement pas d'accepter la tendance à la précarisation des emplois, mais plutôt d'inventer de nouvelles mesures de sécurité sur la base des réalités actuelles du marché du travail et de l'emploi. Il s'agirait en quelque sorte de mettre en place les fondements d'une sécurité ouverte au plus grand nombre et ne reposant plus exclusivement sur le lien d'emploi, mais sur un ensemble d'activités utiles socialement, y compris celle de maintenir ses compétences. L'approche des «marchés transitionnels de travail » nous semble à cet effet per- 
mettre d'assortir le modèle de « l'employabilité » de nouveaux droits pour les travailleurs et travailleuses, droits qu'il reste encore à définir.

Sans doute y aurait-il intérêt à poursuivre cette étude pour voir comment se définiront ces nouveaux droits à travers les actions collectives initiées en région. L'intérêt serait aussi de voir comment les négociations actuelles entre les acteurs, au niveau micro et méso des localités et des régions, pourraient éventuellement déboucher au niveau macro sur l'établissement de nouvelles règles et dispositifs institutionnels concernant les politiques publiques d'emploi et de formation.

\section{Notes et références}

1 Si la coexistence de ces deux phénomènes, à première vue opposés, de chômage et de pénuries de main-d'oeuvre peut paraître paradoxale, elle ne l'est pas. Sans parler de la segmentation des marchés régionaux d'emploi, les pénuries de main-d'œuvre apparaissent en effet le plus souvent dans les territoires comme la conséquence du « cercle vicieux » du sous-développement où les fermetures d'usines se soldent par des phénomènes d'exode des populations et donc de pénuries de main-d'œuvre qui, à leur tour, freineront le développement économique de la région.

2 Cette recherche a été menée pour la Centrale des syndicats du Québec (CSQ) et financée par le Fonds national de formation de la main-d'œuvre (FNFMO).

3 Bernier, C. (dir.) (2004). Dynamiques régionales de développement et formation de la main-d'oeuvre, Montréal, Fonds national de formation de la main-d'œuvre.

4 Pour contrer les pénuries de main-d'œuvre, plusieurs autres actions territoriales prennent d'autres publics pour ci- bles, par exemple les assistés sociaux, les femmes, les immigrants, les jeunes. Pour le propos de cet article, nous nous en tenons ici aux travailleurs en emploi.

5 Boissonat, J. (1995). Le travail dans vingt ans, Plan-Odile Jacob.

6 Supiot, A. (1999). Au-delà de l'emploi, Flammarion.

7 Le rapport principal de recherche a été publié en 2002 : Schmid, G. et B. Gazier (dir.) (2002). The Dynamics of Full Employment, Social Integration through Transitional Labour Markets, Cheltenham (UK) et North Hampton (USA), Edward Elgar; en plus de ce rapport, nous nous servons ici d'un livre publié en français : Gazier, B. (2003). Tous «Sublimes ». Vers un nouveau plein-emploi, Paris, Flammarion, Essais.

8 L'approche des marchés transitionnels est d'ailleurs connectée à une autre approche, celle de la « flexicurité » pour faire le lien entre les besoins de flexibilité et de sécurité (http://www.tilburguniversity.nl/flexicurity).

9

Gazier (2003), op. cit.

10 Osterman, P. et al. (2001). Working in America. A blueprint for the New Labor Market, Cambridge (Massachussets, USA) et Londres (England), The MIT Press.

11 Si le projet peut sembler utopique, il n'est qu'à noter certaines propositions ressorties tout récemment de la commission parlementaire sur la loi du $1 \%$; par exemple, certains rapports patronaux y mettent de l'avant l'idée que l'assurance-emploi pourrait soutenir le financement des personnes qui voudraient se former ou encore, apporter une contribution à la reconnaissance et au bilan des compétences des personnes. Par ailleurs, dans les divers mémoires se dégagea un certain consensus sur la nécessité de promouvoir le regroupement d'entreprises en mutuelles de formation. 